\title{
Structural Analysis of Alkaline-Soluble Polysaccharide, P-1, from the Kernels of Prunus mume SIEB. et ZuCC.
}

\author{
Chikaku Dogasaki, ${ }^{*, a}$ Hajime MurakamI, ${ }^{a}$ Motohiro Nishimima, ${ }^{b}$ Naohito OHNo, ${ }^{c}$ \\ Toshiro YADOMAE, ${ }^{c}$ and Toshio MIYAZAKI ${ }^{c}$ \\ Faculty of Environmental Health, Azabu University, ${ }^{a}$ 1-17-71, Fuchinobe, Sagamihara-shi, Kanagawa 229, Japan, \\ Tokyo Metropolitan Research Laboratory of Public Health, ${ }^{b}$ 3-24-1, Hyakunin-cho, Shinjuku-ku, Tokyo 169. Japan, \\ and Tokyo College of Pharmacy, ${ }^{c}$ Horinouchi, Hachioji-shi, Tokyo 192-03, Japan. \\ Received August 4, 1994; accepted November 10, 1994
}

\begin{abstract}
A polysaccharide fraction extracted with cold $0.5 \mathrm{M} \mathrm{NaOH}$ from the kernels of Prunus mume exhibited some biological activities. A polysaccharide, P-1, was purified from the $0.5 \mathrm{M} \mathrm{NaOH}$ extract by ion-exchange chromatography and gel-filtration. The results of the structural analysis of $P-1$ to determine the relationship between the activities and the structure are described in this paper. In the mild acid hydrolysis of P-1, the nondialyzable hydrolysate (I-3) believed to be its core portion was obtained. The yield of I-3 was $26.0 \%$ and contained $59.8 \%$ uronic acid as galacturonic acid (GalA). The neutral sugars of I-3 were composed of rhamnose, xylose and galactose in a molar ratio of $1.0: 3.4: 0.3$ following analysis by gas-liquid chromatography. The molecular weight of I-3 was estimated to be ca. 14000 by gel-filtration on Toyopearl HW55F. I-3 exhibited the mitogenic activity toward spleen cells as well as P-1. These facts appeared to confirm that I-3 was the core part of P-1 and important for its biological activity. I-3 was successfully reduced by the Taylor and Conrad method to avoid so much repetition. Methylation analysis of the reduced hydrolysate by gas-liquid chromatography and gas chromatography-mass spectroscopy showed that the ratio of 1,4-linked galactopyranosyl and 1,3,4-linked galactopyranosyl residues were significantly increased in comparison with native I-3. These results suggested that I-3 was composed of 1,4- and $1,3,4$-linked galacturonic acid residues in the main chain.
\end{abstract} activity

Key words Prunus mume kernel; polysaccharide; controlled hydrolysis; uronic acid; methylation analysis; mitogenic

We have been studying the biologically-active components of Prunus mume SIEB. et ZuCC. (Japanese name, ume), and earlier described the biological activities of some of the extracts. The hexane extracts obtained from the fruit extract and kernels $P$. mume exhibited the antimutagenic activities. ${ }^{1)}$ Some of the polysaccharide fractions extracted with either water or alkali showed various biological activities, and we also reported the biological activities and the characterization of polysaccharide (P-1) obtained from cold $0.5 \mathrm{M} \mathrm{NaOH}$ extract. $\left.{ }^{2}\right)$

In this paper, we studied the chemical structure of $\mathrm{P}-1$ by a mild hydrolysis with $0.01,0.05$ and $0.1 \mathrm{M}$ trifluoroacetic acid (TFA) to elucidate the relationship between this structure and biological activities.

\section{MATERIALS AND METHODS}

Preparation of Alkaline-Soluble Polysaccharide (P-1) ${ }^{2)}$ The preparation methods of the polysaccharide fractions from the kernels of Prunus mume, a cultivar name of Nanko produced in Mie prefecture, were described previously, and P-1 was isolated from the dialyzable fraction extracted with $0.5 \mathrm{M} \mathrm{NaOH}$ using Toyopearl $650 \mathrm{M}$ ion-exchange chromatography followed by Toyopearl HW65 gel-filtration.

Controlled Hydrolysis P-1 $(500 \mathrm{mg})$ was hydrolyzed with $0.01 \mathrm{M}$ TFA at $100^{\circ} \mathrm{C}$ for $3 \mathrm{~h}$, and dialyzed against distilled water overnight. The dialysis was repeated 2 times. The resulting dialyzable (E-1) and non-dialyzable (I-1) fractions were concentrated and lyophilized. Fraction I-1 was hydrolyzed with $0.05 \mathrm{M}$ TFA, $100^{\circ} \mathrm{C}$ for $3 \mathrm{~h}$ and dialyzed to prepare dialyzable (E-2) and non-dialyzable

* To whom correspondence should be addressed.
(I-2) fractions. Fraction I-2 was again hydrolyzed with $0.1 \mathrm{M}$ TFA, $100^{\circ} \mathrm{C}$ for $3 \mathrm{~h}$ and dialyzed to prepare dialyzable (E-3) and non-dialyzable fractions. The non-dialyzable fraction of the hydrolysate with $0.1 \mathrm{M}$ TFA was designated I-3. A diagram of the controlled hydrolysis is shown in Fig. 1.

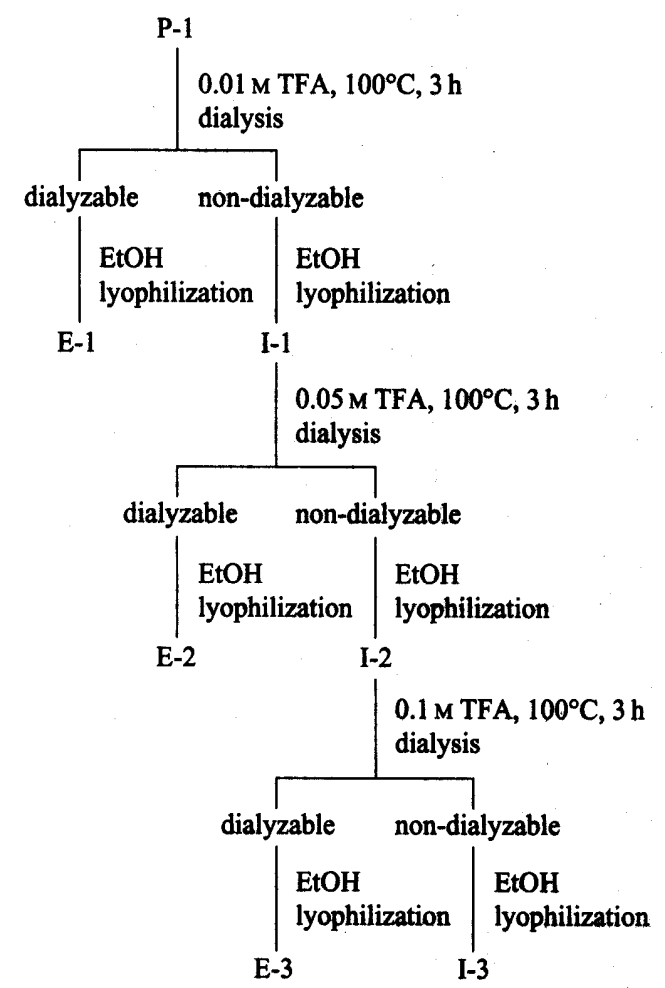

Fig. 1. Diagram of Controlled Hydrolysis Procedure of P-1

(C) 1995 Pharmaceutical Society of Japan 
Physicochemical Analysis The neutral sugar content of I-3 was calculated from the standard sugars, L-rhamnose (L-Rha), D-xylose (D-Xyl), L-arabinose (L-Ara) and D-galactose (D-Gal), mixed according to the molar ratio of I-3 obtained following gas-liquid chromatography (GLC). The uronic acid content of I-3 was calculated from the D-galacturonic acid (D-GalA) standard. Detection of the neutral sugars ${ }^{3)}$ and uronic acid ${ }^{4}$ was carried out using methods described previously.

Analysis of component sugars of I-3 was carried out as follows: I-3 $(5 \mathrm{mg})$ was hydrolyzed with $1 \mathrm{ml} 1 \mathrm{M}$ TFA at $100^{\circ} \mathrm{C}$ for $3 \mathrm{~h}$. The hydrolysate was evaporated to remove TFA and dried under reduced pressure. The residue was dissolved in $0.5 \mathrm{ml}$ of water and divided into two portions. One of them was derivatized to alditol acetates ${ }^{5)}$ in the usual way, and analyzed by GLC using a Shimadzu GC-8A instrument fitted with a glass column $(0.26 \times 200 \mathrm{~cm})$ packed with $3 \%$ ECNSS-M on Gaschrom $Q$ under conditions of $170{ }^{\circ} \mathrm{C}$ column temperature and $60 \mathrm{ml}\left(\mathrm{N}_{2}\right.$ gas)/min flow rate. The other portion of hydrolysate was submitted to thin-layer chromatography (TLC) on an Avicel SF plate $(10 \times 10 \mathrm{~cm})$ and high performance thin-layer chromatography (HPTLC) plate $(10 \times 10 \mathrm{~cm})$. The solvent system used was ethyl acetate-pyridine-acetic acid-water $(5: 5: 1: 3, \mathrm{v} / \mathrm{v})$. Spots were detected by spraying with $p$-anisidine- $\mathrm{HCl}$ reagent. ${ }^{6}{ }^{6}$

The carbon-13 nuclear magnetic resonance $\left({ }^{13} \mathrm{C}-\mathrm{NMR}\right)$ spectral analysis of P-1 and I-3 was carried out using a Bruker AM 400. The spectra were recorded at $27^{\circ} \mathrm{C}$ for solutions in deuterium oxide $\left(\mathrm{D}_{2} \mathrm{O}\right)$, and were obtained in the pulsed Fourier-transform mode with complete proton decoupling. ${ }^{7)}$

The molecular weight of I-3 was measured by gelfiltration using a column of Toyopearl HW $55 \mathrm{~F}$ and eluted with $0.1 \mathrm{M} \mathrm{NaOH}$.

Reduction of Acidic Moieties Uronic acid moieties in I-3 were reduced with $\mathrm{NaBH}_{4}$ by the modified Taylor and Conrad method. ${ }^{8)}$ Briefly, the polysaccharide $(10 \mathrm{mg})$ was dissolved in $10 \mathrm{ml}$ of water containing 1-cyclohexyl-3-(2morpholinoethyl) carbodiimide metho-p-toluenesulfonate (CMC, $50 \mathrm{mg}$ ). As the reaction proceeded, the $\mathrm{pH}$ value of the reaction mixture was maintained at $\mathrm{pH} 4.75$ with $0.1 \mathrm{~m}$ hydrochloric acid. The whole reaction was allowed to proceed for $2 \mathrm{~h}$. After hydrogen ion uptake had ceased, sodium borohydride solution $(800 \mathrm{mg}$ in $10 \mathrm{ml}$ of water) was added gradually to the reaction mixture using a hypodermic syringe; $\mathrm{pH}$ value of the mixture was maintained at 7.0 with $4.0 \mathrm{M}$ hydrochloric acid. The reduction product was isolated by dialysis, then lyophilized and designated I-3R.

Methylation Analysis The reduced product was methylated three times by the method of Hakomori. ${ }^{9)}$ Then, the methylated product was formalized with $90 \%$ formic acid $(1 \mathrm{ml})$ at $100^{\circ} \mathrm{C}$ for $3 \mathrm{~h}$ in a sealed tube. After removal of the formic acid by evaporation, the residue was hydrolyzed with $1 \mathrm{M}$ TFA at $100^{\circ} \mathrm{C}$ for $6 \mathrm{~h}$ followed by evaporation to dryness. The resulting partially $O$-methylated sugars were reduced with sodium borohydride at room temperature for $8 \mathrm{~h}$ to the corresponding alditol, and then derivatized to alditol acetates followed by acetylation. ${ }^{5}$ The resulting products were analyzed by
GLC and gas chromatography-mass spectroscopy (GCMS).

GLC analysis of partially $O$-methylated alditol acetates was performed with a glass column $(0.26 \times 200 \mathrm{~cm})$ packed with 3\% ECNSS-M on Gaschrom Q ( $80-100$ mesh). Flow rate of the carrier gas $\left(\mathrm{N}_{2}\right)$ was $60 \mathrm{ml} / \mathrm{min}$, and the column temperature was $165^{\circ} \mathrm{C}$. The corresponding alditol acetates of $O$-methylated sugars were identified by retention times relative to that of 1,5-di-O-acetyl-2,3,4,6-tetra- $O$ methyl-D-glucitol as $1.0 .^{10)}$

GC-MS analysis of partially $O$-methylated alditol acetates was performed under the following conditions. The gas chromatograph used was a Varian 3400 instrument fitted with a capillary column $(0.025 \mathrm{~mm} \times 30 \mathrm{~m})$ of DB-5, the column oven temperature was $150-200^{\circ} \mathrm{C}$ and carrier gas was $4.0 \mathrm{ml}(\mathrm{He}) / \mathrm{min}$ of the flow rate. The electron impact mass spectra were recorded by a Finnigan MAT INCOS 50 instrument. The ion source temperature was $180^{\circ} \mathrm{C}$ and EI was $70 \mathrm{eV}$.

Biological Activity Mitogen assay was performed to investigate the biological effects on the controlled hydrolysates of $\mathrm{P}-1$ using spleen cells from $\mathrm{C} 3 \mathrm{H} / \mathrm{HeN}$ male mice, followed by assessment using the MTT [3-(4,5dimethylthiazol-2-yl)-2,5-diphenyl tetrazolium bromide ${ }^{11)}$ and alkaline phosphatase (ALP) ${ }^{12)}$ methods as described previously.

\section{RESULTS}

Controlled Hydrolysis of P-1 To gain information about its architecture, P-1 was degraded with mild acid hydrolysis using TFA in the order of $0.01,0.05$ and $0.1 \mathrm{M}$. After each hydrolysate was dialyzed against distilled water, the dialyzable and non-dialyzable fractions were lyophilized respectively. The dialyzable fractions were designated E-1, E-2 and E-3, and then the non-dialyzable fractions were designated I-1, I-2 and I-3. The yield of each fraction is shown in Table I: the yields for dialyzable fractions were $18.8 \%(E-1), 39.8 \%(E-2)$ and $15.4 \%$ (E-3), and the yield for the non-dialyzable fraction (I-3) obtained finally by the partial hydrolysis was $26.0 \%$.

The molar ratio of mono-saccharides in each dialyzable fraction is also shown in Table I. Almost all of the Ara residue contained in P-1 was hydrolyzed under milder conditions, 0.01 or $0.05 \mathrm{M}$ TFA, though Xyl, Gal and uronic acid were gradually hydrolyzed in any concentration of TFA, and existed in the dialyzable fractions; most of the uronic acid remained in the non-dialyzable fraction even under $0.1 \mathrm{M}$ TFA and was contained in I-3 at the rate

TABLE I. Component Sugars of Controlled Hydrolysates of P-1

\begin{tabular}{|c|c|c|c|c|c|c|}
\hline \multirow{2}{*}{ Fraction } & \multirow{2}{*}{$\begin{array}{c}\text { Yield } \\
(\%)\end{array}$} & \multicolumn{4}{|c|}{ Molar ratio of sugars } & \multirow{2}{*}{$\begin{array}{c}\text { GalA } \\
(\%)\end{array}$} \\
\hline & & Rha & Ara & Xyl & Gal & \\
\hline E-1 ${ }^{a)}$ & 18.8 & nd $^{d)}$ & 7.4 & 1.0 & 0.2 & 30.6 \\
\hline $\mathrm{E}-2^{b)}$ & 39.8 & $\mathrm{nd}^{d)}$ & 8.3 & 1.0 & 0.5 & 9.2 \\
\hline $\mathrm{E}-3^{(c)}$ & 15.4 & $\mathrm{nd}^{d)}$ & $\mathrm{nd}^{d)}$ & 1.0 & 0.4 & 26.9 \\
\hline I-3 & 26.0 & 0.3 & $\mathrm{nd}^{\mathrm{d})}$ & 1.0 & 0.1 & 59.8 \\
\hline
\end{tabular}

$a, b, c)$ E-1, E-2 and E-3 are the molar ratios of mono-saccharides contained in dialyzable fractions of controlled hydrolysates. $d$ ) nd: not detected. 
of $c a .60 \%$.

Physicochemical Properties of I-3 I-3 contained 59.8\% of uronic acid (as GalA), and neutral sugars. The component neutral sugars were identified by GLC and TLC as Rha, Xyl and Gal in the molar ratio of approximately $1.0: 3.4: 0.3$. The results suggested that the core portion of P-1 which had molecular weight of over 2000000 was constituted of Rha, Xyl, Gal and GalA in the molar ratio of $1.0: 3.4: 0.3: 7.0$, respectively.

Gel-filtration of I-3 was carried out using Toyopearl

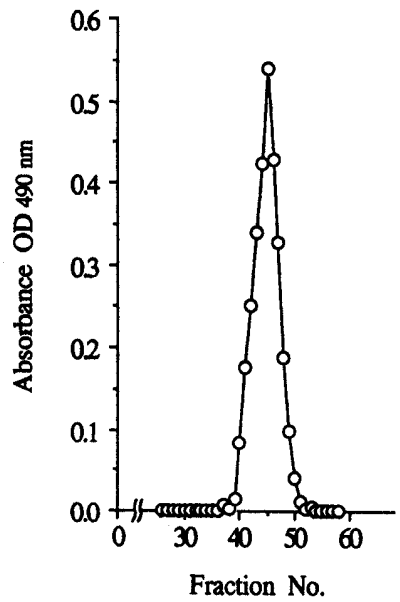

Fig. 2. Chromatogram of I-3 by Gel-Filtration

Ten $\mathrm{mg}$ of I-3 dissolved in $0.5 \mathrm{ml}$ of water was applied to a Toyopearl HW55F column $(2.2 \times 90 \mathrm{~cm})$ and then eluted with $0.1 \mathrm{M} \mathrm{NaOH}$. Eluted fractions were detected individually by the phenol- $\mathrm{H}_{2} \mathrm{SO}_{4}$ method.

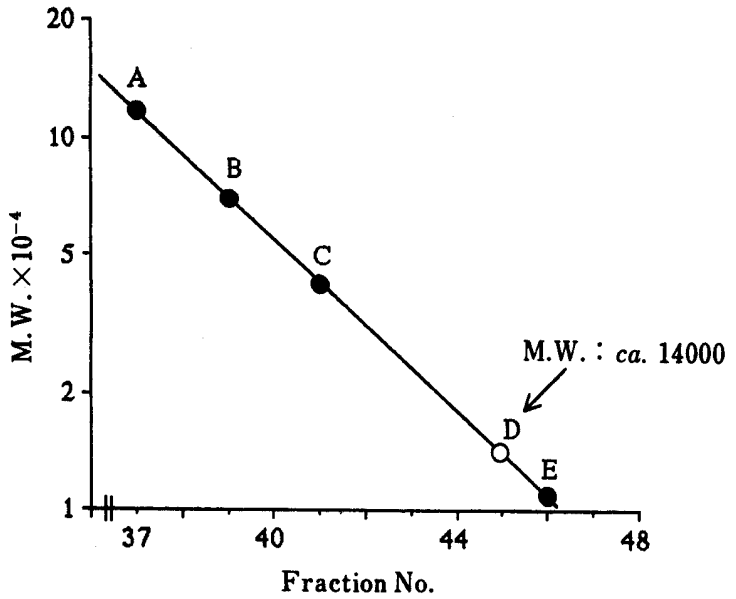

Fig. 3. Determination of Molecular Weight of I-3 by Gel-Filtration on Toyopearl HW55F Eluted with $0.1 \mathrm{M} \mathrm{NaOH}$

A, Dextran T-110; B, Dextran T-70; C, Dextran T-40; D, I-3; E, Dextran T-10.
HW $55 \mathrm{~F}$ column and the chromatogram obtained is shown in Fig. 2. The molecular weight of I-3 was found to be approximately about 14000 by gel-filtration (Fig. 3).

The ${ }^{13}$ C-NMR spectra of P-1 and I-3 are shown in Fig. 4. As shown above, I-3 showed a strong carbonyl signal at $174 \mathrm{ppm}$ suggesting a high content of uronic acid, and this was also confirmed from the signal for galactopyranosyluronic acid $^{13)}$ which appeared at about $100 \mathrm{ppm}$. Comparison of the patterns of P-1 and I-3 showed that most of the signals in I-3 were attributable to minor signals in P-1. Sharp signals in P-1 came from areas around the I-3 core.

Reduction Product of I-3 with $\mathrm{NaBH}_{4}$ The component sugars in the reduction product of I-3, I-3R, were identified as Rha, Xyl and Gal by GLC in the molar ratio of approximately $1.0: 1.5: 4.0$. In comparison with the molar ratio of I-3, Xyl of I-3R was decreased to less than half. Thus, an irregular degradation of Xyl occurred under a condition of severe reduction by $\mathrm{NaBH}_{4}$ and $\mathrm{HCl}$.

Mitogenic Activity of I-3 and I-3R The mitogenic activity against spleen cells of $\mathrm{C} 3 \mathrm{H} / \mathrm{HeN}$ mice was examined to confirm whether or not the controlled hydrolysates of P-1 exhibited biological activity. As shown

TABLE II. Mitogenic Activity of Controlled Hydrolysates towards $\mathrm{C} 3 \mathrm{H} / \mathrm{HeN}$ Spleen Cells ${ }^{a)}$

\begin{tabular}{|c|c|c|c|}
\hline Sample & $\mu \mathrm{g} /$ culture & $\begin{array}{c}\text { MTT } \\
\text { mean } \pm \text { S.D. }{ }^{b)} \text { (S.I.) }\end{array}$ & $\begin{array}{c}\text { ALP } \\
\text { mean } \pm \text { S.D. }{ }^{b)}(\text { S.I. })\end{array}$ \\
\hline Control & & $0.068 \pm 0.0014(1.0)$ & $0.533 \pm 0.0573(1.0)$ \\
\hline \multirow[t]{3}{*}{ P-1 } & 250 & $0.127 \pm 0.0045(1.9)$ & $1.429 \pm 0.1689(2.7)$ \\
\hline & 50 & $0.131 \pm 0.0134(1.9)$ & $0.785 \pm 0.0813(1.5)$ \\
\hline & 10 & $0.106 \pm 0.0088(1.6)$ & $0.563 \pm 0.0702(1.1)$ \\
\hline \multirow[t]{3}{*}{ I-1 } & 250 & $0.111 \pm 0.0091(1.6)$ & $0.999 \pm 0.0952(1.9)$ \\
\hline & 50 & $0.103 \pm 0.0052(1.5)$ & $0.948 \pm 0.0360(1.8)$ \\
\hline & 10 & $0.086 \pm 0.0061(1.3)$ & $0.689 \pm 0.0281(1.3)$ \\
\hline \multirow[t]{3}{*}{$\mathrm{I}-2$} & 250 & $0.138 \pm 0.0016(2.0)$ & $1.100 \pm 0.0579$ \\
\hline & 50 & $0.102 \pm 0.0080(1.5)$ & $0.769 \pm 0.0401(1.4)$ \\
\hline & 10 & $0.089 \pm 0.0117(1.3)$ & $0.723 \pm 0.0130$ \\
\hline \multirow[t]{3}{*}{$\mathrm{I}-3$} & 250 & $0.133 \pm 0.0013(2.0)$ & $1.141 \pm 0.1528$ \\
\hline & 50 & $0.120 \pm 0.0069(1.8)$ & $0.894 \pm 0.0264(1.7)$ \\
\hline & 10 & $0.098 \pm 0.0062(1.4)$ & $0.692 \pm 0.0297(1.3)$ \\
\hline \multirow[t]{3}{*}{ LPS } & 2 & $0.296 \pm 0.0218(4.4)$ & $2.854 \pm 0.0211(5.4)$ \\
\hline & 0.4 & $0.234 \pm 0.0070(3.4)$ & $2.786+0.0415(5.2)$ \\
\hline & 0.08 & $0.159 \pm 0.0110(2.3)$ & $2.157+0.0261(4.0)$ \\
\hline
\end{tabular}

a) $5 \times 10^{5}$ cells $/ 100 \mu \mathrm{l}$ were mixed with $50 \mu \mathrm{l}$ of each sample and $50 \mu \mathrm{l}$ of twofold concentration of RPMI 1640 medium without fetal calf serum, and cultured for $72 \mathrm{~h}$ in humidified $5 \% \mathrm{CO}_{2}$. After being cultured, $25 \mu \mathrm{l}$ of each cultured mixture was transferred to a new tissue culture plate, and MTT and ALP assay was performed. b) The results are expressed as mean O.D. \pm S.D. of triplicate cultures. LPS: lipopolysaccharide.

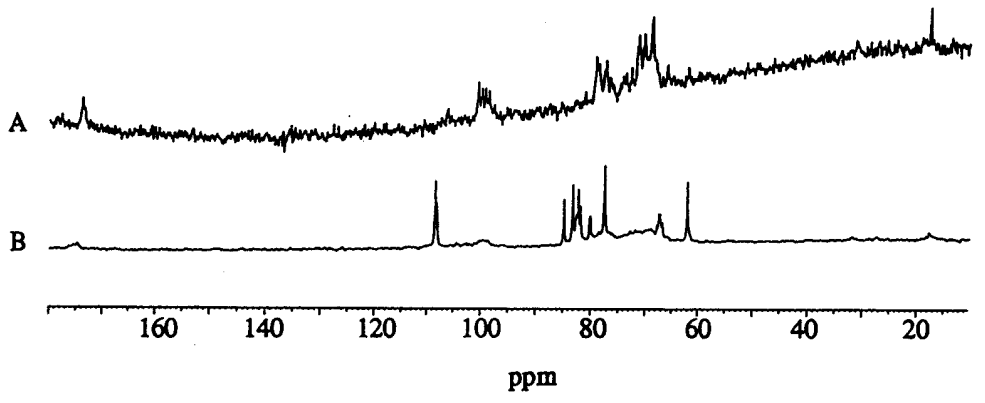

Fig. 4. ${ }^{13} \mathrm{C}-\mathrm{NMR}$ Spectra of $\mathrm{P}-1$ and $\mathrm{I}-3$ in Deuterium Oxide

A, I-3; B, P-1. 
TABLE III. Mitogenic Activity of I-3 and I-3R towards Spleen Cells ${ }^{a)}$

\begin{tabular}{cccc}
\hline Sample & $\mu$ g/culture & $\begin{array}{c}\text { MTT } \\
\text { mean } \pm \text { S.D. }^{b)}(\text { S.I. })\end{array}$ & $\begin{array}{c}\text { ALP } \\
\text { mean } \pm \text { S.D. } .^{b)}(\text { S.I. })\end{array}$ \\
\hline Control & & $0.068 \pm 0.0014(1.0)$ & $0.533 \pm 0.0573(1.0)$ \\
I-3 & 250 & $0.133 \pm 0.0013(2.0)$ & $1.141 \pm 0.1528(2.1)$ \\
& 50 & $0.120 \pm 0.0069(1.8)$ & $0.894 \pm 0.0264(1.7)$ \\
& 10 & $0.098 \pm 0.0062(1.4)$ & $0.692 \pm 0.0297(1.3)$ \\
I-3R & 250 & $0.081 \pm 0.0063(1.1)$ & $0.563 \pm 0.0260(1.1)$ \\
& 50 & $0.076 \pm 0.0024(1.1)$ & $0.580 \pm 0.0120(1.1)$ \\
LPS & 10 & $0.085 \pm 0.0034(1.2)$ & $0.528 \pm 0.0099(1.0)$ \\
& 2 & $0.296 \pm 0.0218(4.4)$ & $2.854 \pm 0.0211(5.4)$ \\
& 0.4 & $0.234 \pm 0.0070(3.4)$ & $2.786 \pm 0.0415(5.2)$ \\
& 0.08 & $0.159 \pm 0.0110(2.3)$ & $2.157 \pm 0.0261(4.0)$ \\
\hline
\end{tabular}

a) Spleen cells $\left(5 \times 10^{5} /\right.$ culture) from $\mathrm{C} 3 \mathrm{H} / \mathrm{HeN}$ mice were mixed with $50 \mu \mathrm{P}-1$ $(5 \mathrm{mg} / \mathrm{ml})$ and cultured without fetal calf serum. After being cultured for $72 \mathrm{~h}$, MTT and ALP assay was performed. b) The results are expresed as mean O.D. \pm S.D. of triplicate cultures.

TABLE IV. Relative Molar Ratio of Alditol Acetates Derived from Methylated I-3 and I-3R

\begin{tabular}{lll}
\hline \multirow{2}{*}{ Component } & \multicolumn{2}{c}{ Molar ratio $^{a)}$} \\
\cline { 2 - 3 } & $\mathrm{I}-3$ & $\mathrm{I}-3 \mathrm{R}$ \\
\hline 2,3,4-Tri- $O$-methyl xylitol & 1.2 & 0.6 \\
3,4-Di- $O$-methyl rhamnitol & 1.0 & 1.0 \\
2,3,4,6-Tetra- $O$-methyl galactitol & 0.5 & 0.3 \\
2,3,6-Tri- $O$-methyl galactitol & $\mathrm{nd}^{b)}$ & 3.2 \\
2,6-Di- $O$-methyl galactitol & $\mathrm{nd}^{b)}$ & 1.4 \\
\hline
\end{tabular}

a) The molar ratios were calculated from mol masses and peak area of derivatives on an ECNSS-M column. b) nd: not detected.

in Table II, all of the hydrolysates, I-1, I-2 and I-3, exhibited similar mitogenic activity to P-1. However, the mitogenic activity of I-3R was not similar to that of I-3 (Table III). These results strongly suggest that I-3 is the core portion of P-1, and that the acidic moieties of I-3 are significant for the mitogenic activity.

Methylation Analysis of I-3 and I-3R The methylated alditol acetates derived from I-3 and I-3R were identified by the retention time of the corresponding alditol acetates relative to that of 1,5 -di- $O$-acetyl-2,3,4,6-tetra- $O$ methyl glucitol on GLC $^{10)}$ and the fragmentation patterns on GC-MS. ${ }^{10,14)}$ The derivatives of 1,5 -di- $O$-acetyl2,3,4-tri- $O$-methyl xylitol, 1,2,5-tri- $O$-acetyl-3,4-di- $O$ methyl rhamnitol and 1,5-di-O-acetyl-2,3,4,6-tetra- $O$ methyl galacutitol were analyzed in both I-3 and I-3R. However, I-3R gave the high peaks of 1,4,5-tri- $O$-acetyl2,3,6-tri- $O$-methyl galacutitol and 1,3,4,5-tri- $O$-acetyl2,6-di- $O$-methyl galacutitol which were not detected in the original I-3. The molar ratios of these derivatives are shown in Table IV. These results strongly suggest that 2,3,6-tri-O-methyl galacutitol and 2,6-di-O-methyl galacutitol increased in methylated I-3R are originated in acidic moieties of I-3, galacturonic acid residues.

\section{DISCUSSION}

It is generally accepted that the mitogenic activity of certain polysaccharides has some correlation with their effectiveness, e.g., prophylactic action against certain etiological agents for human gastro-intestinal diseases.
Therefore, to determine the specific substance(s) effective in promoting human health, mitogenic activity as one of biological activities was tested for the compounds obtained from the kernels of Prunus mume.

Of the six fractions extracted with various solvents from the kernels of $P$. mume, the alkaline-soluble polysaccharide fraction extracted with $0.5 \mathrm{M} \mathrm{NaOH}$ had the highest sugar content. P-1 purified from this polysaccharide fraction was therefore used in further experiments such as the identification and quantification of individual sugar components. Analysis of P-1 fraction by TLC and GLC revealed that the component sugars were mainly Ara and GalA, and included Rha, Xyl and Gal as minor component sugars. In the weak hydrolysis condition with 0.01 and $0.05 \mathrm{~m}$ TFA, primarily Ara was isolated as a mono- and/or oligo-saccharide from the core part. This suggests that Ara is present on the terminal or the tip portion of P-1, and that the architecture of $\mathrm{P}-1$ is an acidic, highly branched heteroglycan-like pectin. The results of the ${ }^{13} \mathrm{C}-\mathrm{NMR}$ spectra of P-1 and I-3 showed most of the signals in I-3 to be minor signals in P-1. I-3 was thus assumed to be the core of P-1.

The data shown in this and a previous paper ${ }^{2)}$ suggested strongly that the mitogen found in $P$. mume has different properties than bacterial lipopolysaccharide, this is shown especially in its reactivity to $\mathrm{C} 3 \mathrm{H} / \mathrm{HeJ}$ mice. A core part given by the partial hydrolysis of P-1 and designated I-3, contained a higher ratio of galacturonic acid. The fact that the activity between P-1 and I-3 resembled each ether suggests strongly that I-3 core part is the essential domain showing mitogenic activity by $\mathrm{P}-1$. That the reduction of GalA in I-3 significantly reduced the activity strongly supported that the GalA part is important for the mitogenic activity of I-3 and P-1. However, commercially available pectin and pectic acid showed almost no mitogenic activity (data not shown). Methylation analysis of I-3R suggested that the major linkage of GalA was $(1 \rightarrow 4)$ and that it was sensitive to pectinase (data not shown). These facts suggested that even though the outline of the linkages of I-3 resembled those of "pectic acid", a certain specific linkage in I-3 is essential to show mitogenic activity.

In comparison with the molar ratio shown in the component sugar analysis and methylation analysis of I-3, one of the component sugars, Xyl, gave a reduced ratio in methylation analysis. Thus, we analyzed the molar ratio of component sugars in I-3 under different hydrolysis conditions: $0.5,1.0$ and $2.0 \mathrm{M} \mathrm{TFA}$ for 5 and $10 \mathrm{~h}$ at $100^{\circ} \mathrm{C}$. Under these conditions, the content of $\mathrm{Xyl}$ was decreased in accordance with increase in the concentration of TFA and the period of hydrolysis (data not shown). Previously, Shimizu et al. ${ }^{15)}$ prepared oligosaccharides from a plant polysaccharide by hydrolysis using $2.0 \mathrm{M}$ TFA at $100^{\circ} \mathrm{C}$ for $3 \mathrm{~h}$. The conditions we used in this report are not significantly stronger than those used in preparing oligosaccharide. However, since I-3 contains Rha residue which is sensitive to acid hydrolysis, we could not choose more severe conditions. I-3 thus appears to contain both acid-sensitive and -resistant glycosidic linkages, so that the molar ratio would contain some inaccuracy. We also used Taylor's reduction, but the reaction sometimes lost a part 
of the component sugars. ${ }^{16)}$ This would explain the contradiction in the molar ratio in the component sugar analysis and the methylation analysis. Although reduction of the activity after Taylor's reduction strongly supported the significance of GalA residue, we could not completely rule out the contribution of another component sugar on the mitogenic activity because the drastic reaction to Taylor's reduction sometimes causes loss of a component, though not a specific component.

Further structural analysis to identify active site of I-3 is worthy investigating.

Acknowledgement The authors are indebted to Mr. Kosai Matsumoto, the president of Ume Kenkyukai Foundation, for his invaluable assistance in providing them with kernels of $P$. mume.

\section{REFERENCES}

1) C. Dogasaki, H. Murakami, M. Nishijima, K. Yamamoto, T. Miyazaki, Yakugaku Zasshi, 112, 577 (1992).
2) C. Dogasaki, H. Murakami, M. Nishijima, N. Ohno, T. Yadomae, T. Miyazaki, Biol. Pharm. Bull., 17, 386 (1994).

3) M. Dubois, K. A. Gilles, J. K. Hamilton, P. A. Rebers, F. Smith, Anal. Chem., 28, 350 (1956).

4) J. T. Galambos, Anal. Biochem., 19, 119 (1967).

5) T. Miyazaki, Y. Naoi, Chem. Pharm. Bull., 22, 2058 (1974).

6) L. Hough, J. K. N. Jones, W. H. Wadman, J. Chem. Soc., 1950, 1702.

7) N. Ohno, I. Suzuki, S. Oikawa, K. Sato, T. Miyazaki, T. Yadomae, Chem. Pharm. Bull., 32, 1142 (1984).

8) R. L. Taylor, H. E. Conrad, Biochemistry, 11, 1383 (1972).

9) S. Hakomori, J. Biochem. (Tokyo), 55, 205 (1964).

10) H. Bjorndal, B. Lindberg, S. Svensson, Acta Chem. Scand., 21, 1801 (1967).

11) I. Sugawara, S. Ishizuka, T. Tsuji, T. Nishiyama, Igaku No Ayumi, 128, 733 (1984).

12) N. Ohno, Y. Arai, I. Suzuki, T. Yadomae, J. Pharmacobio-Dyn., 9, 593 (1986).

13) N. Shimizu, M. Tomoda, Chem. Pharm. Bull., 33, 5539 (1985).

14) H. Bjorndal, C. G. Hellerqvist, B. Lindberg, S. Svensson, Angew. Chem., Int. Ed. Engl., 9, 610 (1970).

15) N. Shimizu, M. Tomoda, R. Gonda, M. Kanari, A. Kubota, A. Kubota, Chem. Pharm. Bull., 37, 3054 (1989).

16) I. R. Siddiqui, P. J. Wood, Carbohydr. Res., 50, 97 (1976). 